\title{
Convexity of Solutions for an Iterative Equation in Banach Spaces
}

\author{
Xiaobing Gong ${ }^{1,2}$ \\ ${ }^{1}$ Key Laboratory of Numerical Simulation of Sichuan Province, Neijiang, Sichuan 641100, China \\ ${ }^{2}$ College of Mathematics and Information Science, Neijiang Normal University, Neijiang, Sichuan 641100, China \\ Correspondence should be addressed to Xiaobing Gong; xbgong@163.com
}

Received 18 March 2013; Accepted 2 August 2013

Academic Editor: Abdelghani Bellouquid

Copyright (c) 2013 Xiaobing Gong. This is an open access article distributed under the Creative Commons Attribution License, which permits unrestricted use, distribution, and reproduction in any medium, provided the original work is properly cited.

By applying Schauder's fixed point theorem we investigate the existence of increasing (decreasing) solutions of the iterative equation $\mathscr{H}(f) \circ f=F$ and further give conditions under which those solutions are convex or concave. As corollaries we obtain results on iterative equation $G\left(f(x), f^{n_{1}}, \ldots, f^{n_{k}}(x)\right)=F(x)$ in Banach spaces, where $n_{1}, n_{2}, \ldots, n_{k} \geq 2$.

\section{Introduction}

Iterative root problem $[1,2]$, being a weak version of the problem of embedding flows, plays an important role in the theory of dynamical systems. As a natural generalization of the iterative root problem, the polynomial-like iterative equation

$$
\lambda_{1} f(x)+\lambda_{2} f^{2}(x)+\cdots+\lambda_{m} f^{m}(x)=F(x), \quad x \in S,
$$

where $S$ is a subset of a linear space over $\mathbb{R}, F: S \rightarrow S$ is a given function, $\lambda_{i} s(i=1, \ldots, m)$ are real constants, $f: S \rightarrow S$ is the unknown function, and $f^{i}$ is the $i$ th iterate of $f$, that is, $f^{i}(x)=f\left(f^{i-1}(x)\right)$ and $f^{0}(x)=x$ for all $x \in S$, is one of the important iterative functional equations $[3,4]$ and was studied in many papers. For $S \subset \mathbb{R}$, while some works (e.g., [5-11]) are contributed to the case of linear $F$, there are many results given to the case of nonlinear $F$, for example, [12, 13] for $m=2$, [14] for general $m$, [15] for smoothness, and [16] for analyticity. Some efforts were also devoted to (1) in high-dimensional spaces such as in $[17,18]$; radially monotonic solutions were discussed in highdimensional Euclidean spaces by properties of orthogonal group in [18], and the existence of convex solutions was proved by introducing a partial order in Banach spaces in [17]. A general iterative equation can be presented as

$$
G\left(f(x), f^{n_{1}}(x), \ldots, f^{n_{k}}(x)\right)=F(x), \quad x \in S,
$$

where $k>0$ and $n_{1}, n_{2}, \ldots, n_{k} \geq 2$. In $1995, C^{0}$ solutions of (2) were discussed in [19], and as continuations of [19], $C^{1}$ and $C^{2}$ solutions were studied in $[20,21]$, respectively, for $S \subset \mathbb{R}$. In 2007, by lifting maps on the unit circle $\mathbb{T}^{1}$ and maps on the torus $\mathbb{T}^{n}$, the existence, uniqueness, and stability of continuous solutions for (2) were proved on the unit circle in [22]. A more general iterative functional equation

$$
\mathscr{H}(f) \circ f=F
$$

was studied in $[23,24]$ in high-dimensional spaces, where $\mathscr{H}$ is a operator. Equation (3) is a generalization of iterative equation (2). In fact, if $\mathscr{H}(f)=G\left(f^{0}, f^{n_{1}-1}, \ldots, f^{n_{k}-1}\right)$, then (3) becomes (2). In [23], the existence of Lipschitzian solutions for (3) was proved on a compact convex subset of $\mathbb{R}^{N}$, and by using this result, the existence of Lipschitzian solutions for equation

$$
\sum_{n=1}^{+\infty} \lambda_{n} f^{n}(x)=F(x)
$$

was investigated on a compact interval of $\mathbb{R}$ and a compact convex subset of $\mathbb{R}^{N}, N>1$. Later, the results were partially generalized to an arbitrary closed (not necessarily convex) 
subset of a Banach space and the existence of solutions for iterative functional equations

$$
\begin{gathered}
\sum_{n=-\infty}^{+\infty} A_{n} f^{n}(x)=F(x), \\
A_{0} f(x)+\sum_{n=1}^{+\infty} A_{n} f^{n}(x)=F(x),
\end{gathered}
$$

was proved in [24], where $A_{n}$ s are bounded linear operators on the Banach space.

Convexity is an important property of functions and the study of convexity for iterative equations can be traced to 1968 when Kuczma and Smajdor [25] investigated the convexity of iterative roots. Some recent results can be found from $[17,26-$ $28]$. In $[27,28]$, convexity of solutions for (1) was discussed on a compact interval, and in [26], nondecreasing convex solutions for (1) on open intervals were discussed. In [17], convexity of solutions for (1) was studied in Banach spaces. Up to now, there are no further results on monotonicity and convexity of solutions for (2) and (3) in Banach spaces. In fact, there are much more difficulties on monotonicity and convexity of solutions for these two equations in Banach spaces.

In this paper we study monotonicity and convexity of solutions for (2) and (3) in Banach spaces and generalize the results in [17]. Using Schauder's fixed point theorem, we discuss increasing (decreasing) solutions for (3) and further give conditions under which those solutions are convex or concave. As corollaries, we obtain results on (2). The uniqueness and continuous dependence of those solutions are also discussed.

\section{Preliminaries}

As in [17], in order to discuss monotonicity and convexity of solutions in Banach spaces, we need to introduce a partial order. For convenience, we use the conventions of [17]. As in [29], a nonempty subset $K$ of a real vector space $X$ is called a cone if $x \in K$ implies that $a x \in K$ for all $a>0$. A nonempty and nontrivial $(\neq\{\theta\}$, where $\theta$ denotes the zero element of $X)$ subset $K \subset X$ is called an order cone in $X$ if $K$ is a convex cone and satisfies $K \cap(-K)=\{\theta\}$. Having chosen such an order cone $K$ in $X$, we can define a partial order $x \leq_{K} y$ in $X$, simply called the $K$-order, if

$$
y-x \in K \text {. }
$$

A real vector space $X$ equipped with a $K$-order is called an ordered vector space, abbreviated by OVS and denoted by $(X, K)$. A real Banach space $(X,\|\cdot\|)$ associated with a $K$ order is called an ordered real Banach space, abbreviated by OBS and denoted by $(X, K,\|\cdot\|)$, if $K$ is closed. One can define increasing (decreasing) operators as in [30] in an ordered real vector space $(X, K)$. An operator $f: D \subset X \rightarrow X$ is said to be increasing (resp., decreasing) in the sense of the $K$-order if $x \leq_{K} y$ implies $f(x) \leq_{K} f(y)$ (resp., $f(x) \geq_{K} f(y)$ ). An operator $f: D \rightarrow X$, where $D \subset X$ is a convex subset, is said to be convex (resp., concave) in the sense of the $K$ order if $f(\lambda x+(1-\lambda) y) \leq_{K} \lambda f(x)+(1-\lambda) f(y)$ (resp., $\left.f(\lambda x+(1-\lambda) y) \geq_{K} \lambda f(x)+(1-\lambda) f(y)\right)$ for all $\lambda \in[0,1]$ and for every pair of distinct comparable points $x, y \in D$ (i.e., either $x \leq_{K} y$ or $x \geq_{K} y$ ).

Let $\Omega$ be a compact convex subset of an ordered real Banach space $(X, K,\|\cdot\|)$ with nonempty interior, and let $C(\Omega, X)$ consist of all continuous functions $f: \Omega \rightarrow$ $X$. $C(\Omega, X)$ is a Banach space equipped with the norm $\|f\|_{C(\Omega, X)}:=\sup _{x \in \Omega}\|f(x)\|$. For $0 \leq m \leq M<+\infty$, define

$$
\begin{aligned}
E^{+}(\Omega, m, M):= & \{f \in C(\Omega, X): m(y-x) \\
& \leq_{K} f(y)-f(x) \\
& \leq_{K} M(y-x) \text { if } x \leq_{K} y, \\
& \|f(y)-f(x)\| \leq M\|y-x\|
\end{aligned}
$$

if $x$ and $y$ are not comparable ,

$$
\begin{aligned}
E^{-}(\Omega, m, M):= & \{f \in C(\Omega, X): m(y-x) \\
& \leq_{K} f(x)-f(y) \\
& \leq_{K} M(y-x) \text { if } x \leq_{K} y, \\
& \|f(y)-f(x)\| \leq M\|y-x\|
\end{aligned}
$$

if $x$ and $y$ are not comparable ,

$$
\begin{aligned}
& E_{\mathrm{cv}}^{+}(\Omega, m, M):=\left\{f \in E^{+}(\Omega, m, M): f\right. \text { is convex on } \\
&\Omega \text { in } K \text {-order }\}, \\
& E_{\mathrm{cc}}^{+}(\Omega, m, M):=\left\{f \in E^{+}(\Omega, m, M): f\right. \text { is concave } \\
&\text { on } \Omega \text { in } K \text {-order }\},
\end{aligned}
$$

$$
\begin{aligned}
& C^{+}(\Omega, m, M):=\left\{f \in E^{+}(\Omega, m, M): f(\Omega) \subset \Omega\right\}, \\
& C^{-}(\Omega, m, M):=\left\{f \in E^{-}(\Omega, m, M): f(\Omega) \subset \Omega\right\}, \\
& C_{\mathrm{cv}}^{+}(\Omega, m, M):=\left\{f \in E_{\mathrm{cv}}^{+}(\Omega, m, M): f(\Omega) \subset \Omega\right\}, \\
& C_{\mathrm{cc}}^{+}(\Omega, m, M):=\left\{f \in E_{\mathrm{cc}}^{+}(\Omega, m, M): f(\Omega) \subset \Omega\right\}, \\
& I(f):=\inf \left\{M: \forall x, y \in \Omega, \theta \leq_{K} f(y)-f(x)\right. \\
& \leq_{K} M(y-x) \text { if } x \leq_{K} y, \\
&\|f(y)-f(x)\| \leq M\|y-x\|
\end{aligned}
$$

if $x$ and $y$ are not comparable\},

$$
\begin{aligned}
D(f):=\inf \{ & \left\{M: \forall x, y \in \Omega, \theta \leq_{K} f(x)-f(y)\right. \\
& \leq_{K} M(y-x) \text { if } x \leq_{K} y, \\
& \|f(y)-f(x)\| \leq M\|y-x\|
\end{aligned}
$$

if $x$ and $y$ are not comparable .

Similar to Lemma 2.2 in [17], $C^{+}(\Omega, m, M), C^{-}(\Omega, m, M)$, $C_{\mathrm{cv}}^{+}(\Omega, m, M)$, and $C_{\mathrm{cc}}^{+}(\Omega, m, M)$ are compact convex subsets of $C(\Omega, X)$. 
As shown in $[29,30]$, an order cone $K$ in an ordered real Banach space $(X,\|\cdot\|)$ is said to be normal if there exists a constant $N>0$ such that $\|x\| \leq N\|y\|$ if $\theta \leq_{K} x \leq_{K} y$ in $X$. The smallest constant $N$, denoted by $N(K)$, is called the normal constant of $K$.

\section{Main Result}

We first discuss monotonicity and convexity of solutions for iterative functional equation (3) in the ordered real Banach space $(X, K,\|\cdot\|)$ such that $K$ is normal and $N(K) \leq 1$. Consider (3) with the following hypothesis:

(H1) $\mathscr{H}(f)(x)=x-\mathscr{P}(f)(x)$.

\subsection{Increasing and Decreasing Solutions}

Theorem 1. Suppose that (H1) holds and $F \in E^{+}\left(\Omega, 0, p_{1}\right)$, where $p_{1} \in(0,+\infty)$ is a constant. Let $\mathscr{P}: C^{+}(\Omega, 0, \infty) \rightarrow$ $E^{+}(\Omega, 0, \infty)$ such that

$$
\mathscr{P}(f) \in E^{+}(\Omega, 0, \alpha(I(f))),
$$

where $\alpha:(0,+\infty) \rightarrow(0,+\infty)$ is an increasing function and $F(x)+\mathscr{P}(f)(y) \in \Omega$ for any $x, y \in \Omega$. If there exists $p \in$ $(0, \infty)$ such that

$$
p_{1} \leq p(1-\alpha(p))
$$

and $\left.\mathscr{P}\right|_{C^{+}(\Omega, 0, p)}$ is continuous, then (3) has a solution $f \in$ $C^{+}(\Omega, 0, p)$.

Proof. Define a mapping $L: C^{+}(\Omega, 0, p) \rightarrow C(\Omega, X)$ by

$$
L f(x)=F(x)+\mathscr{P}(f) \circ f(x) .
$$

We first prove that $L$ is a self-mapping on $C^{+}(\Omega, 0, p)$. Obviously, $\mathscr{P}(f) \circ f$ is well defined and $F+\mathscr{P}(f) \circ f \in$ $C(\Omega, X)$. By $F(x)+\mathscr{P}(f)(y) \in \Omega$ for any $x, y \in \Omega$ and the definition of $C^{+}(\Omega, 0, p),(F+\mathscr{P}(f) \circ f)(\Omega) \subset \Omega$. Further, when $x, y \in \Omega$ are not comparable, that is, $x-y \notin K$ and $y-x \notin K$, by (8) and $F \in E^{+}\left(\Omega, 0, p_{1}\right)$, we have

$$
\begin{aligned}
& \|L f(x)-L f(y)\| \\
& \quad=\|(F(x)-F(y))+(\mathscr{P}(f) \circ f(x)-\mathscr{P}(f) \circ f(y))\| \\
& \quad \leq\|F(x)-F(y)\|+\|\mathscr{P}(f) \circ f(x)-\mathscr{P}(f) \circ f(y)\| \\
& \quad \leq p_{1}\|x-y\|+\alpha(I(f))\|f(x)-f(y)\| \\
& \quad \leq\left(p_{1}+p \alpha(p)\right)\|x-y\|,
\end{aligned}
$$

where the monotonicity of the function $\alpha$ is employed, which implies that

$$
\|L f(x)-L f(y)\| \leq p\|x-y\|
$$

because of (9). When $x, y \in \Omega$ are comparable, suppose that $x \leq_{K} y$. By the definition of $C^{+}(\Omega, 0, p), \theta \leq_{K} f(y)-$ $f(x) \leq_{K} p(y-x)$; thus $f(x) \leq_{K} f(y)$. Hence, by (8), we get

$$
\begin{aligned}
\theta & \leq_{K} \mathscr{P}(f) \circ f(y)-\mathscr{P}(f) \circ f(x) \\
& \leq_{K} \alpha(I(f))(f(y)-f(x)) .
\end{aligned}
$$

Consequently, we have

$$
\begin{aligned}
\theta & \leq_{K} L f(y)-L f(x) \\
& =(F(y)-F(x))+(\mathscr{P}(f) \circ f(y)-\mathscr{P}(f) \circ f(x)) \\
& \leq p_{1}(y-x)+\alpha(I(f))(f(y)-f(x)) \\
& \leq\left(p_{1}+p \alpha(p)\right)(y-x),
\end{aligned}
$$

where the monotonicity of the function $\alpha$ is employed, which implies that

$$
\theta \leq_{K} L f(y)-L f(x) \leq_{K} p(y-x)
$$

because of (9). Thus, (12) and (15) imply that $L$ is a selfmapping on $C^{+}(\Omega, 0, p)$. The continuity of $\left.\mathscr{P}\right|_{C^{+}(\Omega, 0, p)}$ implies that $L$ is continuous on $C^{+}(\Omega, 0, p)$. Since $C^{+}(\Omega, 0, p)$ is a compact convex subset, by Schauder's fixed point theorem, we see that $L$ has a fixed point $f \in C^{+}(\Omega, 0, p)$. Thus, $f$ is an increasing solution of (3). The proof is completed.

The following is devoted to decreasing solutions.

Theorem 2. Suppose that (H1) holds and $F \in E^{-}\left(\Omega, 0, p_{1}\right)$. Let $\mathscr{P}: C^{-}(\Omega, 0, \infty) \rightarrow E^{+}(\Omega, 0, \infty)$ such that

$$
\mathscr{P}(f) \in E^{+}(\Omega, 0, \alpha(I(f))),
$$

where $\alpha:(0,+\infty) \rightarrow(0,+\infty)$ is an increasing function and $F(x)+\mathscr{P}(f)(y) \in \Omega$ for any $x, y \in \Omega$. If condition (9) holds for a constant $p \in(0, \infty)$ and $\left.\mathscr{P}\right|_{C^{-}(\Omega, 0, p)}$ is continuous, then (3) has a solution $f \in C^{-}(\Omega, 0, p)$.

The proof is almost the same as the proof of Theorem 1; we omit it here.

3.2. Convex and Concave Solutions. On the basis of the last subsection, we can discuss convexity of solutions for (3).

Theorem 3. Suppose that (H1) holds and $F \in E_{c v}^{+}\left(\Omega, 0, p_{1}\right)$, where $p_{1} \in(0,+\infty)$ is a constant. Let $\mathscr{P}: C_{c v}^{+}(\Omega, 0, \infty) \rightarrow$ $E_{c v}^{+}(\Omega, 0, \infty)$ such that

$$
\mathscr{P}(f) \in E_{c v}^{+}(\Omega, 0, \alpha(I(f))),
$$

where $\alpha:(0,+\infty) \rightarrow(0,+\infty)$ is an increasing function and $F(x)+\mathscr{P}(f)(y) \in \Omega$ for any $x, y \in \Omega$. If there exists $p \in(0, \infty)$ such that

$$
p_{1} \leq p(1-\alpha(p))
$$

and $\left.\mathscr{P}\right|_{C_{c v}^{+}(\Omega, 0, p)}$ is continuous, then (3) has a solution $f \in$ $C_{c v}^{+}(\Omega, 0, p)$.

In order to prove Theorem 3, we need the following lemma.

Lemma 4 (see $[17$, Lemma 3.1]). Let $(X, K,\|\cdot\|)$ be an ordered real Banach space. Then composition $f \circ g$ is convex (resp., concave) if both $f$ and $g$ are convex (resp., concave) and increasing. In particular, for increasing convex (resp., concave) operator $f$, the iterate $f^{k}$ is also convex (resp., concave). 
Proof of Theorem 3. Define a mapping $L: C_{\mathrm{cv}}^{+}(\Omega, 0, p) \rightarrow$ $C(\Omega, X)$ as in Theorem 1 . In order to prove that $L$ is a selfmapping on $C_{\mathrm{cv}}^{+}(\Omega, 0, p)$, it suffices to prove that $F+\mathscr{P}(f) \circ f$ is convex in the sense of $K$-order on $\Omega$. In fact, by (17), we know $\mathscr{P}(f)(x)$ is increasing and convex on $\Omega$. Hence, by Lemma 4 and $f \in C_{\mathrm{cv}}^{+}(\Omega, 0, p), \mathscr{P}(f) \circ f$ is convex in the sense of $K$ order on $\Omega$. Consequently, by $F \in E_{\mathrm{cv}}^{+}\left(\Omega, 0, p_{1}\right)$,

$$
\begin{aligned}
F(t x+ & (1-t) y)+\mathscr{P}(f) \circ f(t x+(1-t) y) \\
\leq & t F(x)+(1-t) F(y) \\
& +t \mathscr{P}(f) \circ f(x)+(1-t) \mathscr{P}(f) \circ f(y) \\
= & t(F(x)+\mathscr{P}(f) \circ f(x)) \\
& +(1-t)(F(y)+\mathscr{P}(f) \circ f(y))
\end{aligned}
$$

for every pair of distinct comparable points $x, y \in \Omega$ and $t \epsilon$ $[0,1]$. So $L$ is an self-mapping on $C_{\mathrm{cv}}^{+}(\Omega, 0, p)$. The remaining part of the proof is the same as the proof of Theorem 1 . This completes the proof.

Similarly, we can prove the following results for concavity of solutions.

Theorem 5. Suppose that (H1) holds and $F \in E_{c c}^{+}\left(\Omega, 0, p_{1}\right)$, where $p_{1} \in(0,+\infty)$ is a constant. Let $\mathscr{P}: C_{c c}^{+}(\Omega, 0, \infty) \rightarrow$ $E_{c c}^{+}(\Omega, 0, \infty)$ such that

$$
\mathscr{P}(f) \in E_{c c}^{+}(\Omega, 0, \alpha(I(f)))
$$

where $\alpha:(0,+\infty) \rightarrow(0,+\infty)$ is a increasing function and $F(x)+\mathscr{P}(f)(y) \in \Omega$ for any $x, y \in \Omega$. If there exists $p \in(0, \infty)$ such that

$$
p_{1} \leq p(1-\alpha(p))
$$

and $\left.\mathscr{P}\right|_{C_{c c}^{+}(\Omega, 0, p)}$ is continuous, then (3) has a solution $f \in$ $C_{c c}^{+}(\Omega, 0, p)$.

\section{Iterative Equation in Banach Spaces}

In this section, we are going to discuss monotonicity and convexity of solutions for (2) in the ordered real Banach space $(X, K,\|\cdot\|)$ such that $K$ is normal and $N(K) \leq 1$. Consider (2) with the following hypotheses:

(H2) $G\left(y_{0}, y_{1}, \ldots, y_{k}\right)=y_{0}+g\left(y_{1}, \ldots, y_{k}\right), g\left(y_{1}, \ldots, y_{k}\right) \in$ $C\left(\Omega^{k}, X\right)$.

Before discussing convexity, we prove the existence of increasing and decreasing solutions of (2).

4.1. Increasing and Decreasing Solutions. We first study increasing solutions. Consider (2) with the following hypothesis:
(H3) $\exists \beta_{i}>0$ such that

$$
\begin{aligned}
& \theta \leq_{K} g\left(\bar{y}_{1}, \ldots, \bar{y}_{k}\right)-g\left(y_{1}, \ldots, y_{k}\right) \\
& \quad \leq_{K} \sum_{i=1}^{k} \beta_{i}\left(y_{i}-\bar{y}_{i}\right) \quad \text { if } \bar{y}_{i} \leq_{K} y_{i}, \\
& \left\|g\left(y_{1}, \ldots, y_{k}\right)-g\left(\bar{y}_{1}, \ldots, \bar{y}_{k}\right)\right\| \\
& \quad \leq \sum_{i=1}^{k} \beta_{i}\left\|y_{i}-\bar{y}_{i}\right\| \quad \text { for any } y_{i}, \bar{y}_{i} \in \Omega .
\end{aligned}
$$

Theorem 6. Suppose that (H2) and (H3) hold and $F \in$ $E^{+}\left(\Omega, 0, M_{1}\right)$, where $M_{1} \in(0,+\infty)$ is a constant. If $F(x)-$ $g\left(y_{1}, \ldots, y_{k}\right) \in \Omega$ for any $x, y_{i} \in \Omega$ and

$$
M_{1} \leq M-\sum_{i=1}^{k} \beta_{i} M^{n_{i}}
$$

for a constant $M \in(0,+\infty)$, then (2) has a solution $f \in$ $C^{+}(\Omega, 0, M)$. Additionally, if

$$
\sum_{i=1}^{k} \beta_{i} \sum_{j=0}^{n_{i}-1} M^{j}<1
$$

then the solution $f$ is unique in $C^{+}(\Omega, 0, M)$ and depends continuously on F.

In order to prove Theorem 6, we need the following lemmas.

Lemma 7 (see [17, Lemma 3.2]). Let $(X, K,\|\cdot\|)$ be an ordered real Banach space such that $K$ is normal, and let $f, g \in C^{+}(\Omega, m, M)$ (resp., $C^{-}(\Omega, m, M), C_{c v}^{+}(\Omega, m, M)$, and $\left.C_{c c}^{+}(\Omega, m, M)\right)$, where $0 \leq m \leq M \leq+\infty$. Then

$$
\begin{array}{r}
\left\|f^{k}-g^{k}\right\|_{C(\Omega, X)} \leq \sum_{j=0}^{k-1} M_{0}^{j}\|f-g\|_{C(\Omega, X)}, \\
\forall k=1,2, \ldots
\end{array}
$$

Lemma 8 (see [17, Lemma 3.3]). Let $(X, K,\|\cdot\|)$ be an ordered real Banach space, and let $f \in C^{-}(\Omega, m, M)$, where $0 \leq m \leq$ $M<+\infty$. Then

$$
\begin{aligned}
-M^{2 n-1}(y-x) & \leq_{K} f^{2 n-1}(y)-f^{2 n-1}(x) \\
& \leq_{K}-m^{2 n-1}(y-x), \quad n=1,2, \ldots, \\
m^{2 n}(y-x) & \leq_{K} f^{2 n}(y)-f^{2 n}(x) \\
& \leq_{K} M^{2 n}(y-x), \quad n=1,2, \ldots,
\end{aligned}
$$

for all $x \leq_{K} y$ in $\Omega$.

Proof of Theorem 6. We apply Theorem 1. Define

$$
\mathscr{P}(f)(x)=-g\left(f^{n_{1}-1}(x), \ldots, f^{n_{k}-1}(x)\right) .
$$


By $(\mathrm{H} 2), \mathscr{P}(f) \in C(\Omega, X)$. Next we prove that $\mathscr{P}(f)$ is an operator from $C^{+}(\Omega, 0, \infty)$ to $E^{+}(\Omega, 0, \infty)$ such that

$$
\mathscr{P}(f) \in E^{+}(\Omega, 0, \alpha(I(f))) .
$$

By the definition of $C^{+}(\Omega, 0, \infty)$, for $f \in C^{+}(\Omega, 0, \infty)$, we have

$$
\theta \leq_{K} f(y)-f(x) \leq_{K} I(f)(y-x)
$$

if $x \leq_{K} y$, and

$$
\|f(x)-f(y)\| \leq I(f)\|x-y\|
$$

if $x, y \in \Omega$ are not comparable, that is, $x-y \notin K$ and $y-x \notin K$. Note that

$$
\begin{aligned}
\theta & \leq_{K} f^{n_{i}-1}(y)-f^{n_{i}-1}(x) \\
& \leq_{K} I(f)^{n_{i}-1}(y-x), \quad i=1,2, \ldots, k
\end{aligned}
$$

if $x \leq_{K} y$, and

$$
\left\|f^{n_{i}-1}(x)-f^{n_{i}-1}(y)\right\| \leq I(f)^{n_{i}-1}\|x-y\|, \quad i=1,2, \ldots, k
$$

if $x, y \in \Omega$ are not comparable, that is, $x-y \notin K$ and $y-x \notin K$. By (H3), we get

$$
\begin{aligned}
\theta \leq_{K} \mathscr{P}(f)(y)-\mathscr{P}(f)(x)= & g\left(f^{n_{1}-1}(x), \ldots, f^{n_{k}-1}(x)\right) \\
& -g\left(f^{n_{1}-1}(y), \ldots, f^{n_{k}-1}(y)\right) \\
& \leq_{K} \sum_{i=1}^{k} \beta_{i}\left(f^{n_{i}-1}(y)-f^{n_{i}-1}(x)\right) \\
& \leq_{K} \sum_{i=1}^{k} \beta_{i} I(f)^{n_{i}-1}(y-x)
\end{aligned}
$$

if $x \leq_{K} y$, and

$$
\begin{aligned}
\|\mathscr{P}(f)(y)-\mathscr{P}(f)(x)\|= & \| g\left(f^{n_{1}-1}(x), \ldots, f^{n_{k}-1}(x)\right) \\
& -g\left(f^{n_{1}-1}(y), \ldots, f^{n_{k}-1}(y)\right) \| \\
\leq & \sum_{i=1}^{k} \beta_{i}\left\|f^{n_{i}-1}(y)-f^{n_{i}-1}(x)\right\| \\
\leq & \sum_{i=1}^{k} \beta_{i} I(f)^{n_{i}-1}\|y-x\| \\
\leq & \sum_{i=1}^{k} \beta_{i} I(f)^{n_{i}-1}\|y-x\|
\end{aligned}
$$

if $x, y \in \Omega$ are not comparable, that is, $x-y \notin K$ and $y-x \notin K$, where $N(K) \leq 1$ is employed. Hence, $\mathscr{P}(f) \in$
$E^{+}\left(\Omega, 0, \sum_{i=1}^{k} \beta_{i} I(f)^{n_{i}-1}\right)$. Let $\alpha(I(f))=\sum_{i=1}^{k} \beta_{i} I(f)^{n_{i}-1}$. Obviously, function $\alpha$ is increasing on $(0,+\infty)$. By the definition of $C^{+}(\Omega, 0,+\infty)$, we have $f^{n_{i}-1}(x) \in \Omega, i=1,2, \ldots, k$ for any $f \in C^{+}(\Omega, 0,+\infty)$. Hence, $F(x)+\mathscr{P}(f)(y) \in$ $\Omega$, for all $x, y \in \Omega$.

Let $p=M$, and let $p_{1}=M_{1}$; by (23),

$$
\begin{aligned}
p(1-\alpha(p)) & =M\left(1-\sum_{i=1}^{k} \beta_{i} M^{n_{i}-1}\right) \\
& =M-\sum_{i=1}^{k} \beta_{i} M^{n_{i}} \geq M_{1} .
\end{aligned}
$$

Next, we prove that $\left.\mathscr{P}(f)\right|_{C^{+}(\Omega, 0, M)}$ is continuous. By (H3), for any $f_{1}, f_{2} \in C^{+}(\Omega, 0, M)$, by Lemma 7 , we have

$$
\begin{aligned}
& \left\|\mathscr{P}\left(f_{1}\right)-\mathscr{P}\left(f_{2}\right)\right\|_{C^{+}(\Omega, X)} \\
& =\sup _{x \in \Omega} \| g\left(f_{1}^{n_{1}-1}(x), f_{1}^{n_{2}-1}(x), \ldots, f_{1}^{n_{k}-1}(x)\right) \\
& \quad-g\left(f_{2}^{n_{1}-1}(x), f_{2}^{n_{2}-1}(x), \ldots, f_{2}^{n_{k}-1}(x)\right) \| \\
& \leq \sum_{i=1}^{k} \beta_{i}\left\|f_{1}^{n_{i}-1}(x)-f_{2}^{n_{i}-1}(x)\right\|_{C^{+}(\Omega, X)} \\
& \leq \sum_{i=1}^{k} \beta_{i} \sum_{j=0}^{n_{i}-2} M^{j}\left\|f_{1}-f_{2}\right\|_{C(\Omega, X)} .
\end{aligned}
$$

By Theorem 1 , there exists an $f \in C^{+}(\Omega, 0, M)$ such that

$$
\mathscr{H}(f) \circ f=F .
$$

By (H2), we have

$$
G\left(f, f^{n_{1}}, \ldots, f^{n_{k}}\right)=F .
$$

This completes the proof of existence. Under the additional hypothesis (24), we see from (36) that the mapping $L$ defined as (10) is a contraction mapping on the closed subset $C^{+}(\Omega, 0, M)$. The uniqueness of solution follows. Let $f_{1}, f_{2} \in$ $C^{+}(\Omega, 0, M)$ be solutions of (2) with the given functions $F_{1}, F_{2}$ respectively. By the uniqueness, $f_{1}=F_{1}+\mathscr{P}\left(f_{1}\right) \circ f_{1}$ and $f_{2}=F_{2}+\mathscr{P}\left(f_{2}\right) \circ f_{2}$. Hence,

$$
\begin{aligned}
\| f_{1} & -f_{2} \|_{C(\Omega, X)} \\
= & \left\|\left(F_{1}-F_{2}\right)+\left(\mathscr{P}\left(f_{1}\right) \circ f_{1}-\mathscr{P}\left(f_{2}\right) \circ f_{2}\right)\right\|_{C(\Omega, X)} \\
\leq & \left\|F_{1}-F_{2}\right\|_{C(\Omega, X)}+\left\|\mathscr{P}\left(f_{1}\right) \circ f_{1}-\mathscr{P}\left(f_{2}\right) \circ f_{2}\right\|_{C(\Omega, X)} \\
\leq & \sum_{i=1}^{k} \beta_{i} \sum_{j=0}^{n_{i}-1} M^{j}\left\|f_{1}-f_{2}\right\|_{C(\Omega, X)} \\
& +\left\|F_{1}-F_{2}\right\|_{C(\Omega, X)} .
\end{aligned}
$$

By (24), we have $\left\|f_{1}-f_{2}\right\|_{C(\Omega, X)} \leq \quad(1 /(1-$ $\left.\left.\sum_{i=1}^{k} \beta_{i} \sum_{j=0}^{n_{i}-1} M^{j}\right)\right)\left\|F_{1}-F_{2}\right\|_{C(\Omega, X)}$, implying that the solution of (2) depends continuously on $F$. The proof is completed. 
Similarly, we can prove the following result for decreasing solutions. We need the following hypothesis:

(H4) $\exists \beta_{i}>0$ such that

$$
\begin{aligned}
& \theta \leq_{K} g\left(y_{1}, \ldots, y_{k}\right)-g\left(\bar{y}_{1}, \ldots, \bar{y}_{k}\right) \\
& \quad \leq_{K} \sum_{i=1}^{k} \beta_{i}\left(y_{i}-\bar{y}_{i}\right) \quad \text { if } \bar{y}_{i} \leq_{K} y_{i}, \\
& \left\|g\left(y_{1}, \ldots, y_{k}\right)-g\left(\bar{y}_{1}, \ldots, \bar{y}_{k}\right)\right\| \\
& \quad \leq \sum_{i=1}^{k} \beta_{i}\left\|y_{i}-\bar{y}_{i}\right\| \quad \text { for any } y_{i}, \bar{y}_{i} \in \Omega .
\end{aligned}
$$

Theorem 9. Suppose that (H2) and (H4) hold and all even order iterates in (2) vanish. Let $F \in E^{-}\left(\Omega, 0, M_{1}\right)$, where $M_{1} \in(0,+\infty)$ is a constant. If $F(x)-g\left(y_{1}, \ldots, y_{k}\right) \in \Omega$, for all $x, y_{i} \in \Omega$ and

$$
M_{1} \leq M-\sum_{i=1}^{k} \beta_{i} M^{n_{i}}
$$

for a constant $M \in(0,+\infty)$, then (2) has a solution $f \in$ $C^{-}(\Omega, 0, M)$. Additionally, if

$$
\sum_{i=1}^{k} \beta_{i} \sum_{j=0}^{n_{i}-1} M^{j}<1,
$$

then the solution $f$ is unique in $C^{-}(\Omega, 0, M)$ and depends continuously on F.

By using Lemma 8, the proof is almost the same as the proof of Theorem 6 . We omit it here.

4.2. Convex and Concave Solutions. On the basis of the last subsection we can discuss convexity of solutions for (2). Consider (2) with the following hypothesis:

(H5) if $y_{i} \leq_{K} \bar{y}_{i}$ or $\bar{y}_{i} \leq_{K} y_{i}$ in $\Omega$, then

$$
\begin{aligned}
& \operatorname{tg}\left(y_{1}, \ldots, y_{k}\right)+(1-t) g\left(\bar{y}_{1}, \ldots, \bar{y}_{k}\right) \\
& \quad \leq_{K} g\left(t y_{1}+(1-t) \bar{y}_{1}, \ldots, t y_{k}+(1-t) \bar{y}_{k}\right) .
\end{aligned}
$$

Theorem 10. Suppose that (H2), (H3), and (H5) hold and $F \in$ $E_{c v}^{+}\left(\Omega, 0, M_{1}\right)$, where $M_{1} \in(0,+\infty)$ is a constant. If $F(x)-$ $g\left(y_{1}, \ldots, y_{k}\right) \in \Omega$ for any $x, y_{i} \in \Omega$ and

$$
M_{1} \leq M-\sum_{i=1}^{k} \beta_{i} M^{n_{i}}
$$

for a constant $M \in(0,+\infty)$, then (2) has a solution $f \in$ $C_{c v}^{+}(\Omega, 0, M)$. Additionally, if

$$
\sum_{i=1}^{k} \beta_{i} \sum_{j=0}^{n_{i}-1} M^{j}<1,
$$

then the solution $f$ is unique in $C_{c v}^{+}(\Omega, 0, M)$ and depends continuously on F.
Proof. Similar to Theorem 6, by applying Theorem 3, it suffices to prove that $\mathscr{P}(f):=-g\left(f^{n_{1}-1}, \ldots, f^{n_{k}-1}\right)$ is convex in the sense of $K$-order. In fact, each $f^{n_{i}-1}, i=1,2, \ldots, k$ is convex in the sense of $K$-order because $f$ is increasing and convex by Lemma 4 . Hence, for every distinct comparable point $x, y \in \Omega$, suppose $x \leq_{K} y$; by (H3) and (H5), we have

$$
\begin{gathered}
\mathscr{P}(f)(t x+(1-t) y) \\
=-g\left(f^{n_{1}-1}(t x+(1-t) y), \ldots,\right. \\
\left.f^{n_{k}-1}(t x+(1-t) y)\right) \\
\leq_{K}-g\left(t f^{n_{1}-1}(x)+(1-t) f^{n_{1}-1}(y), \ldots,\right. \\
\left.\quad t f^{n_{k}-1}(x)+(1-t) f^{n_{k}-1}(y)\right) \\
\leq_{K}-t g\left(f^{n_{1}-1}(x), \ldots, f^{n_{k}-1}(x)\right) \\
-(1-t) g\left(f^{n_{1}-1}(y), \ldots, f^{n_{k}-1}(y)\right) \\
=t \mathscr{P}(f)(x)+(1-t) \mathscr{P}(f)(y) .
\end{gathered}
$$

The proof is completed.

Similarly, we can prove the following results for concavity of solutions. We need the following hypothesis:

(H6) if $y_{i} \leq_{K} \bar{y}_{i}$ or $\bar{y}_{i} \leq_{K} y_{i}$ in $\Omega$, then

$$
\begin{aligned}
& g\left(t y_{1}+(1-t) \bar{y}_{1}, \ldots, t y_{k}+(1-t) \bar{y}_{k}\right) \\
& \quad \leq_{K} t g\left(y_{1}, \ldots, y_{k}\right)+(1-t) g\left(\bar{y}_{1}, \ldots, \bar{y}_{k}\right) .
\end{aligned}
$$

Theorem 11. Suppose that (H2), (H3), and (H6) hold and $F \in$ $E_{c c}^{+}\left(\Omega, 0, M_{1}\right)$, where $M_{1} \in(0,+\infty)$ is a constant. If $F(x)-$ $g\left(y_{1}, \ldots, y_{k}\right) \in \Omega$ for any $x, y_{i} \in \Omega$ and

$$
M_{1} \leq M-\sum_{i=1}^{k} \beta_{i} M^{n_{i}}
$$

for a constant $M \in(0,+\infty)$, then (2) has a solution $f \in$ $C_{c v}^{+}(\Omega, 0, M)$. Additionally, if

$$
\sum_{i=1}^{k} \beta_{i} \sum_{j=0}^{n_{i}-1} M^{j}<1,
$$

then the solution $f$ is unique in $C_{c v}^{+}(\Omega, 0, M)$ and depends continuously on $F$.

Example 12. Let $X=C([0,1], \mathbb{R})$ equipped with the norm $\|x\|=\sup _{t \in[0,1]}|x(t)|$ for $x \in X$. Let

$$
\begin{aligned}
\Omega:= & \left\{x \in C([0,1],[0,1]):\left|x\left(t_{1}\right)-x\left(t_{2}\right)\right|\right. \\
& \left.\leq\left|t_{1}-t_{2}\right|, t_{1}, t_{2} \in[0,1]\right\},
\end{aligned}
$$

a subset of $X$. Then, the equation

$$
\begin{aligned}
& G\left(f(x), f^{2}(x), f^{3}(x)\right) \\
& \quad=f(x)-\frac{1}{32}\left(f^{2}(x)\right)^{2}-\frac{1}{48} e^{f^{3}(x)}=\frac{1}{4} x^{2}, \quad \forall x \in \Omega,
\end{aligned}
$$


is an iterative equation of the form (2) in the infinitedimensional setting, where

$$
G\left(y_{0}, y_{1}, y_{2}\right)=y_{0}-\frac{1}{32} y_{1}^{2}-\frac{1}{48} e^{y_{2}}
$$

and $F(x):=(1 / 4) x^{2}$. Note that

$$
K:=\mathbb{R}_{+}^{[0,1]}:=\{x \in C([0,1], \mathbb{R}): x(t) \geq 0\}
$$

is a normal order cone in $X$ and $N(K)=1$. Then, $\Omega$ is a compact convex subset of the ordered real Banach space $(X, K,\|\cdot\|)$. Clearly, $(\mathrm{H} 2)$ is satisfied and

$$
g\left(y_{1}, y_{2}\right)=-\frac{1}{32} y_{1}^{2}-\frac{1}{48} e^{y_{2}}
$$

If $\bar{y}_{1} \leq_{K} y_{1}, \bar{y}_{2} \leq_{K} y_{2}, \bar{y}_{i}, y_{i} \in \Omega, i=1,2$, then

$$
\begin{aligned}
0 & \leq_{K} g\left(\bar{y}_{1}, \bar{y}_{2}\right)-g\left(y_{1}, y_{2}\right) \\
& =\left(\frac{1}{32} y_{1}^{2}+\frac{1}{48} e^{y_{2}}\right)-\left(\frac{1}{32} \bar{y}_{1}^{2}+\frac{1}{48} e^{\bar{y}_{2}}\right) \\
& \leq_{K} \frac{1}{16}\left(y_{1}-\bar{y}_{1}\right)+\frac{1}{16}\left(y_{2}-\bar{y}_{2}\right) .
\end{aligned}
$$

For any $\bar{y}_{i}, y_{i} \in \Omega$,

$$
\begin{aligned}
& \left\|g\left(y_{1}, y_{2}\right)-g\left(\bar{y}_{1}, \bar{y}\right)\right\| \\
& \quad=\left\|\left(\frac{1}{32} \bar{y}_{1}^{2}+\frac{1}{48} e^{\bar{y}_{2}}\right)-\left(\frac{1}{32} y_{1}^{2}+\frac{1}{48} e^{y_{2}}\right)\right\| \\
& \quad \leq \frac{1}{16}\left\|y_{1}-\bar{y}_{1}\right\|+\frac{1}{16}\left\|y_{2}-\bar{y}_{2}\right\|,
\end{aligned}
$$

where $\beta_{1}=1 / 16, \beta_{2}=1 / 16$. Hence, $(\mathrm{H} 3)$ is satisfied.

$$
\begin{aligned}
& \text { If } y_{i} \leq_{K} \bar{y}_{i} \text { or } \bar{y}_{i} \leq_{K} y_{i} \text { in } \Omega \text {, then } \\
& \begin{aligned}
\operatorname{tg}\left(y_{1}, y_{2}\right)+(1-t) g\left(\bar{y}_{1}, \bar{y}_{2}\right) \\
\quad=t\left(-\frac{1}{32} y_{1}^{2}-\frac{1}{48} e^{y_{2}}\right)+(1-t)\left(-\frac{1}{32} \bar{y}_{1}^{2}-\frac{1}{48} e^{\bar{y}_{2}}\right) \\
\quad=-\frac{1}{32}\left(t y_{1}^{2}+(1-t) \bar{y}_{1}^{2}\right)-\frac{1}{48}\left(t e^{y_{2}}+(1-t) e^{\bar{y}_{2}}\right) \\
\quad \leq_{K}-\frac{1}{32}\left(t y_{1}+(1-t) \bar{y}_{1}\right)^{2}-\frac{1}{48} e^{t y_{2}+(1-t) \bar{y}_{2}} \\
\quad \leq_{K} g\left(t y_{1}+(1-t) \bar{y}_{1}, t y_{2}+(1-t) \bar{y}_{2}\right) .
\end{aligned}
\end{aligned}
$$

Hence, (H5) is satisfied. Similar to Example 4.2 in [17], $F \in$ $E_{\mathrm{cv}}^{+}(\Omega, 0,1 / 2) . F(x)-g\left(y_{1}, y_{2}\right)=(1 / 4) x^{2}+(1 / 32) y_{1}^{2}+$ $(1 / 48) e^{y_{2}} \in \Omega$ for any $x, y_{1}, y_{2} \in \Omega$. In this case,

$$
M-\sum_{i=1}^{k} \beta_{i} M^{n_{i}}-M_{1}=M-\frac{1}{16} M^{2}-\frac{1}{16} M^{3}-\frac{1}{2} \geq 0
$$

for all $M \in[1,3]$; that is, inequality (44) holds. By Theorem 10, (51) has an increasing solution $f \in C_{\mathrm{cv}}^{+}(\Omega, 0, M)$.
Additionally, if $M=1$, we have

$$
\sum_{i=1}^{K} \beta_{i} \sum_{j=0}^{n_{j}-1} M^{j}=\frac{1}{16}(1+M)+\frac{1}{16}\left(1+M+M^{2}\right)=\frac{5}{16}<1 .
$$

Hence, the solution is unique and depends continuously on F.

It is difficult to discuss the convexity of solutions for (2) without hypothesis (H2) and (3) without hypothesis (H1) because of the difficulties in discussing the inverse of a mapping in Banach spaces. The reason also leads to difficulties in discussing the convexity of solutions for (2) and (3) by applying the method in [23].

\section{Acknowledgments}

The author is grateful to the editor and the referee for their valuable comments and encouragement. This work is supported by Key Project of Sichuan Provincial Department of Education (12ZA086).

\section{References}

[1] M. Kuczma, B. Choczewski, and R. Ger, Iterative Functional Equations, vol. 32 of Encyclopedia of Mathematics and its Applications, Cambridge University Press, Cambridge, UK, 1990.

[2] G. Targoński, Topics in Iteration Theory, vol. 6, Vandenhoeck \& Ruprecht, Göttingen, Germany, 1981.

[3] K. Baron and W. Jarczyk, "Recent results on functional equations in a single variable, perspectives and open problems," Aequationes Mathematicae, vol. 61, no. 1-2, pp. 1-48, 2001.

[4] J. Zhang, L. Yang, and W. Zhang, "Some advances on functional equations," Advances in Mathematics, vol. 24, no. 5, pp. 385-405, 1995.

[5] J. G. Dhombres, "Itération linéaire d’ordre deux," Publicationes Mathematicae Debrecen, vol. 24, no. 3-4, pp. 177-187, 1977.

[6] W. Jarczyk, "On an equation of linear iteration," Aequationes Mathematicae, vol. 51, no. 3, pp. 303-310, 1996.

[7] J. Matkowski and W. Zhang, "On linear dependence of iterates," Journal of Applied Analysis, vol. 6, no. 1, pp. 149-157, 2000.

[8] A. Mukherjea and J. S. Ratti, "On a functional equation involving iterates of a bijection on the unit interval," Nonlinear Analysis, vol. 7, no. 8, pp. 899-908, 1983.

[9] A. Mukherjea and J. S. Ratti, "A functional equation involving iterates of a bijection on the unit interval. II," Nonlinear Analysis: Theory, Methods \& Applications, vol. 31, no. 3-4, pp. 459-464, 1998.

[10] J. Tabor and J. Tabor, "On a linear iterative equation," Results in Mathematics, vol. 27, no. 3-4, pp. 412-421, 1995.

[11] D. Yang and W. Zhang, "Characteristic solutions of polynomiallike iterative equations," Aequationes Mathematicae, vol. 67, no. 1-2, pp. 80-105, 2004.

[12] M. Malenica, "On the solutions of the functional equation $\phi(x)+\phi^{2}(x)=F(x)$," Matematički Vesnik, vol. 6, no. 3, pp. 301305, 1982.

[13] L. R. Zhao, "Existence and uniqueness theorem for the solution of the functional equation $\lambda_{1} f(x)+\lambda_{2} f^{[2]}(x)=F(x)$," Journal of China University of Science and Technology, vol. 13, pp. 21-27, 1983 (Chinese). 
[14] W. Zhang, "Discussion on the iterated equationn $\sum_{i=1}^{n} \lambda_{i} f^{i}(x)=$ $F(x)$," Chinese Science Bulletin, vol. 32, pp. 1444-1451, 1987.

[15] W. N. Zhang, "Discussion on the differentiable solutions of the iterated equation $\sum_{i=1}^{n} \lambda_{i} f^{i}(x)=F(x)$," Nonlinear Analysis: Theory, Methods \& Applications, vol. 15, no. 4, pp. 387-398, 1990.

[16] J. G. Si, "The existence of local analytic solutions to the iterated equation $\sum_{i=1}^{n} \lambda_{i} f^{i}(x)=F(x)$," Acta Mathematica Sinica, vol. 37, no. 5, pp. 590-600, 1994 (Chinese).

[17] X. Gong and W. Zhang, "Convex solutions of the polynomiallike iterative equation in Banach spaces," Publicationes Mathematicae Debrecen, vol. 82, no. 2, pp. 341-358, 2013.

[18] W. Zhang, "Solutions of equivariance for a polynomial-like iterative equation," Proceedings of the Royal Society of Edinburgh A, vol. 130, no. 5, pp. 1153-1163, 2000.

[19] J. Si, "Discussion on the $C^{0}$ solution of the iterated equation $G\left(f^{n 0}(x) ; f^{n 1}(x), \ldots, f^{n k}(x)\right)=F(x)$," Journal of Mathematical Research and Exposition, vol. 15, pp. 149-150, 1995 (Chinese).

[20] J. G. Si, " $C^{1}$-solutions of a class of iterative equations," Acta Mathematica Sinica. Chinese Series, vol. 39, no. 2, pp. 247-256, 1996 (Chinese).

[21] J. G. Si and W. N. Zhang, "On $C^{2}$ solutions of a functional equations," Acta Mathematica Sinica, vol. 41, no. 5, pp. 10611064, 1998 (Chinese).

[22] M. C. Zdun and W. Zhang, "A general class of iterative equations on the unit circle," Czechoslovak Mathematical Journal, vol. 57, no. 3, pp. 809-829, 2007.

[23] M. Kulczycki and J. Tabor, "Iterative functional equations in the class of Lipschitz functions," Aequationes Mathematicae, vol. 64, no. 1-2, pp. 24-33, 2002.

[24] J. Tabor and M. Żołdak, "Iterative equations in Banach spaces," Journal of Mathematical Analysis and Applications, vol. 299, no. 2, pp. 651-662, 2004.

[25] M. Kuczma and A. Smajdor, "Fractional iteration in the class of convex functions," Bulletin de l'Académie Polonaise des Sciences. Série des Sciences Mathématiques, Astronomiques et Physiques, vol. 16, pp. 717-720, 1968.

[26] T. Trif, "Convex solutions to polynomial-like iterative equations on open intervals," Aequationes Mathematicae, vol. 79, no. 3, pp. 315-325, 2010.

[27] B. Xu and W. Zhang, "Decreasing solutions and convex solutions of the polynomial-like iterative equation," Journal of Mathematical Analysis and Applications, vol. 329, no. 1, pp. 483497, 2007.

[28] W. Zhang, K. Nikodem, and B. Xu, "Convex solutions of polynomial-like iterative equations," Journal of Mathematical Analysis and Applications, vol. 315, no. 1, pp. 29-40, 2006.

[29] E. Zeidler, Nonlinear Functional Analysis and Its Applications. Vol. I: Fixed-Point Theorems, Springer, New York, NY, USA, 1986, Translated by P. R. Wadsack.

[30] H. Amann, "Fixed point equations and nonlinear eigenvalue problems in ordered Banach spaces," SIAM Review, vol. 18, no. 4, pp. 620-709, 1976. 


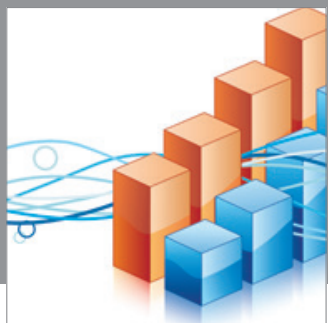

Advances in

Operations Research

mansans

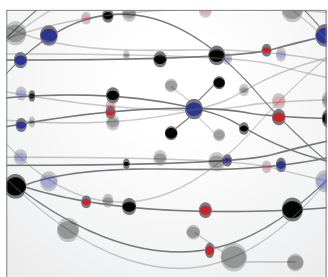

The Scientific World Journal
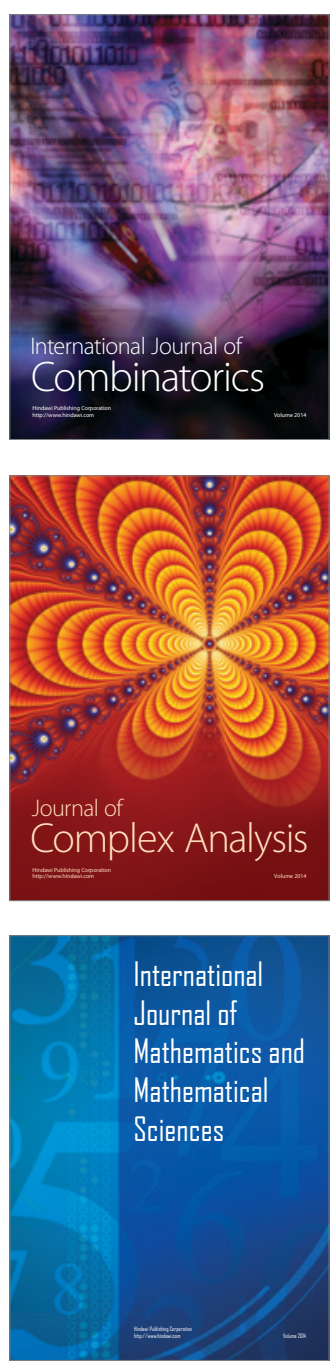
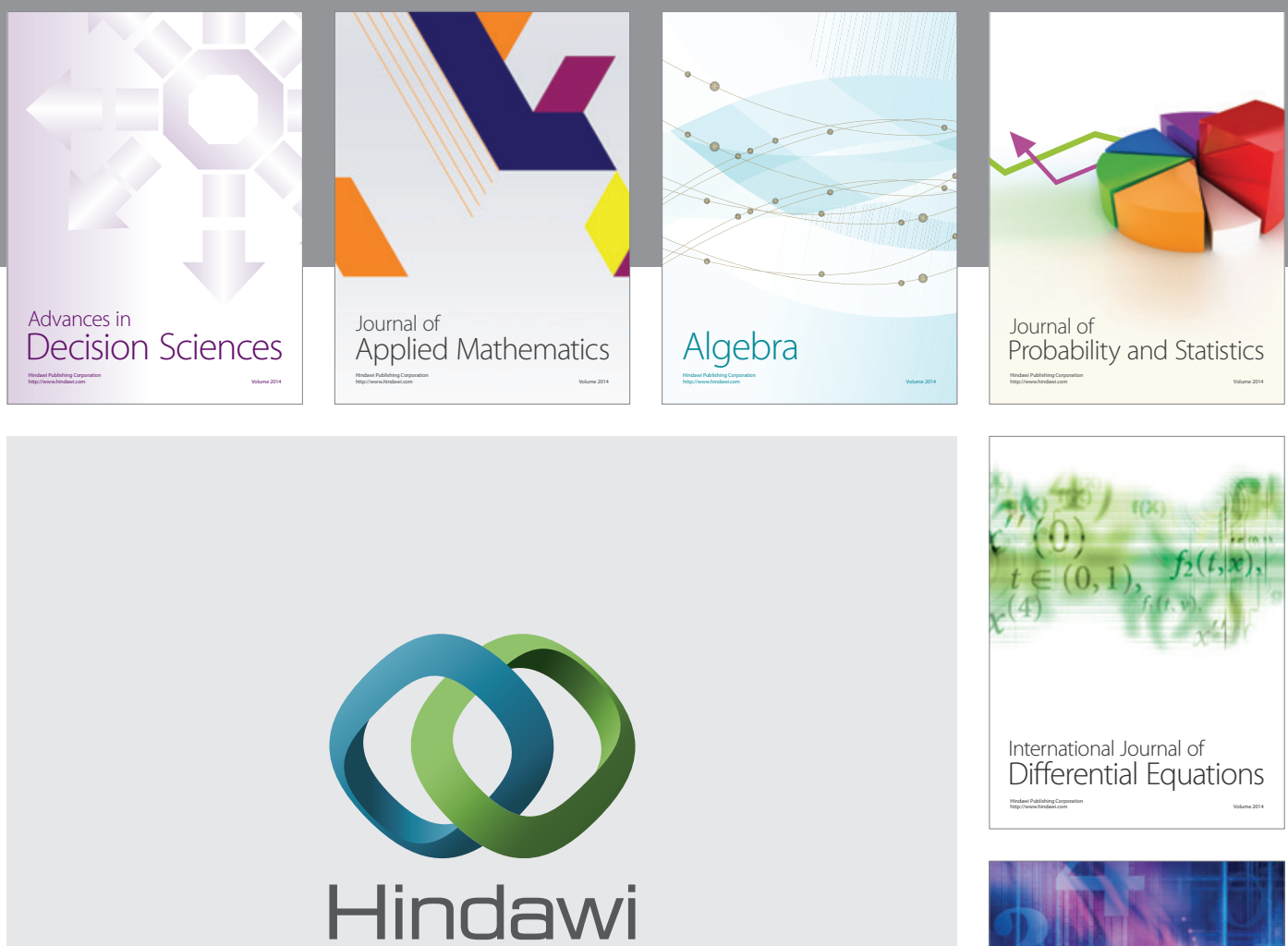

Submit your manuscripts at http://www.hindawi.com
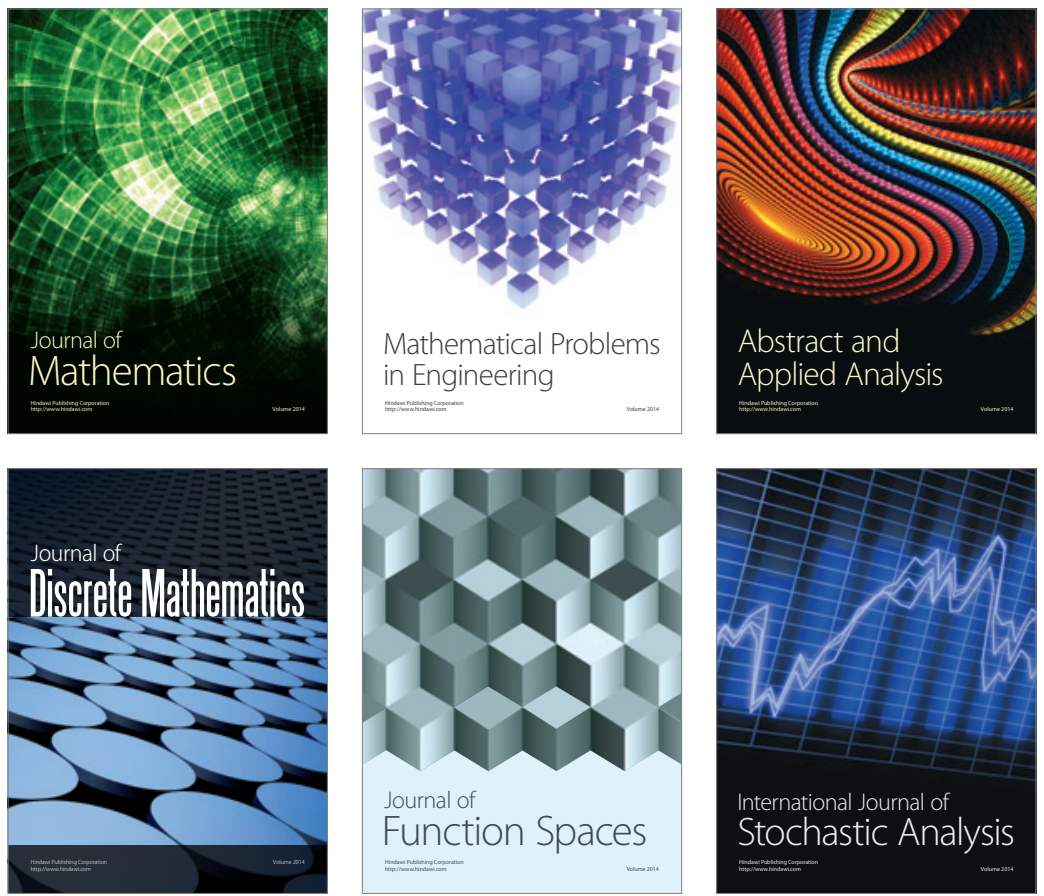

Journal of

Function Spaces

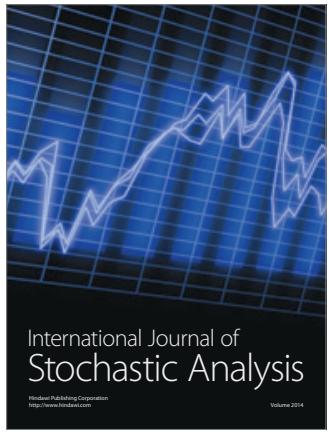

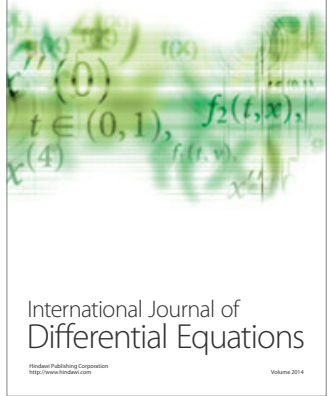
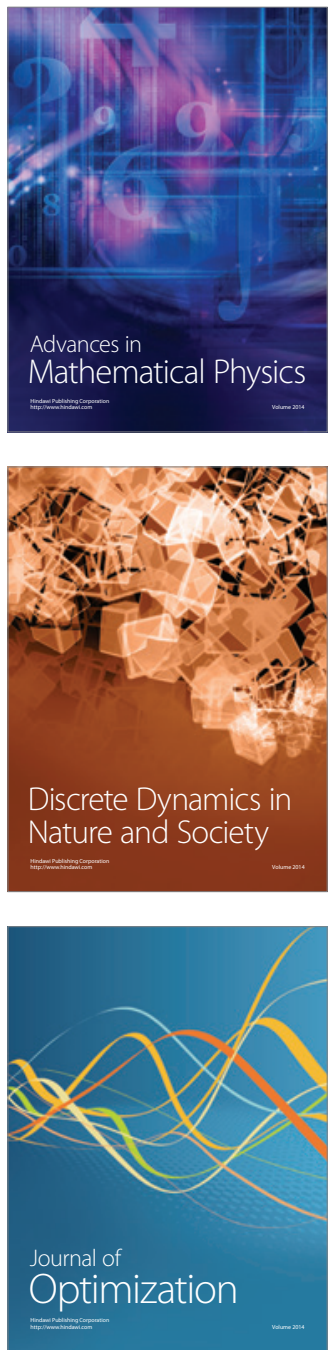